\title{
Manejo forestal sustentable de los recursos no maderables en el semidesierto del norte de México
}

\section{Sustainable forest management of non-wood resources in the semi- desert of northern Mexico}

\author{
Eulalia Edith Villavicencio-Gutiérrez ${ }^{1}$, Antonio Cano-Pineda ${ }^{1}$, David Castillo- \\ Quiroz ${ }^{1}$, Adrián Hernández-Ramos ${ }^{1 *}$ y Oscar Ulises Martínez-Burciaga ${ }^{1+}$
}

\begin{abstract}
The semi-arid ecosystems of northern Mexico cover 70.79 million ha. These are characterized by their high vulnerability to desertification and by the presence of extremely marginalized rural populations, who exploit the non-wood forest resources (NWFR) of various species for the purpose of obtaining raw materials. The objective was to describe the research carried out in 35 years since the creation of the Instituto Nacional de Investigaciones Forestales, Agrícolas y Pecuarias (INIFAP) (National Institute for Research on Forest, Agriculture and Livestock) (INIFAP) regarding the management, use and conservation of the NWFR of northern Mexico for the purpose of providing information to decision makers in public policies aimed at promoting sustainable rural development. In order to describe the research carried out, a search was carried out in the digital scientific sources and physical libraries of INIFAP; the information was then integrated, and the progress made in the several research programs of this Institute was described by species. Some of the topics that have been developed in relation to the NWFR are harvesting practices, sampling systems, forest biometrics, modeling of productive potential, land use planning, selection of vegetative material, seed management, production and quality of the plant in the nursery and greenhouse, establishment and management of plantations, and ex situ conservation of plant genetic resources of native species. These results make it possible to conserve these forest resources and increase their production and productivity, as well as to promote the sustainability of non-timber resources.
\end{abstract}

Key words: Allometry, exploitation, bioenergy, biotechnology, semi-arid ecosystems, plantations.

\section{Resumen}

Los ecosistemas semiáridos del norte de México abarcan 70.79 millones de hectáreas; se caracterizan por su alta vulnerabilidad a la desertificación y la presencia de población rural en extrema marginación, quienes aprovechan los recursos forestales no maderables (RFNM) de diversas especies para obtener materia prima. El objetivo de la presente revisión fue describir las investigaciones realizadas en los 35 años de existencia del Instituto Nacional de Investigaciones Forestales, Agrícolas y Pecuarias (INIFAP) referentes al manejo, aprovechamiento y conservación de los RFNM del norte de México para proporcionar información a los tomadores de decisiones en políticas públicas encauzadas a promover el desarrollo rural sustentable. Para la descripción de las investigaciones desarrolladas, se realizó una búsqueda en fuentes científicas digitales y bibliotecas físicas del INIFAP, enseguida se integró la información y se describió por especie el avance realizado en los diferentes programas de investigación del Instituto. Entre los temas que se han desarrollado están las prácticas de aprovechamiento, sistemas de muestreo, biometría forestal, modelación del potencial productivo, ordenamiento territorial, selección de material vegetativo, manejo de semilla, producción y calidad de planta en vivero e invernadero, establecimiento y manejo de plantaciones, así como conservación ex situ de recursos fitogenéticos de especies nativas. Estos resultados permiten conservar, aumentar la producción y productividad de los RFNM; además de promover la sustentabilidad de los recursos no maderables.

Palabras clave: Alometría, aprovechamiento, bioenergía, biotecnología, ecosistemas semiáridos, plantaciones.

Fecha de recepción/Reception date: 22 de febrero de 2021

Fecha de aceptación/Acceptance date: 9 de julio de 2021

Instituto Nacional de Investigaciones Forestales, Agrícolas y Pecuarias. Campo Experimental Saltillo. México.

*Autor para correspondencia; correo-e: hernandez.adrian@inifap.gob.mx 


\section{Introducción}

Los recursos forestales no maderables (RFNM) de los ecosistemas semiáridos de México aportan $9.4 \%$ de la producción nacional, y es la zona noreste del país la de mayor importancia en ese rubro (Semarnat, 2020). En esta región habita una población rural superior a 5 millones de habitantes, constituida por ejidatarios, comuneros y pequeños propietarios en su mayoría afectados por la pobreza extrema y alta migración, quienes tienen en los RFNM su medio de vida, por lo que influyen en su seguridad alimentaria, salud, bienestar e ingresos económicos (Inegi, 2020).

En México, la producción acumulada de los RFNM en los últimos 30 años es de 844620 t; al respecto, sobresale Coahuila como la entidad con mayor producción acumulada (280 680 t), seguida de Tamaulipas (191 270 t) y Zacatecas (155 850 t) (Semarnat, 2020). En esas entidades y en Chihuahua, Nuevo León y San Luís Potosí las principales especies aprovechadas son la Euphorbia antisyphilitica Zucc. (candelilla), Agave lechuguilla Torr. (lechuguilla), Yucca carnerosana Trel. (yuca), Lippia graveolens HBK. (orégano) y Nolina cespitifera Trel. (cortadillo) (Martínez, 2013). Otros taxones de menor producción son Jatropha dioica Sessé ex Cerv. (sangre de drago), Agave spp. (maguey), Dasylirion spp. (sotol), cactáceas de ornato, plantas aromáticas [Litsea parvifolia (Hemsl.) Mez] y Prosopis spp. (mezquite) (Martínez, 2013).

Dada la importancia de los RFNM en el norte de México es necesario realizar un manejo sustentable de sus poblaciones; para ello, se requiere de una planificación integrada, en la cual el manejo sostenible debe considerar los componentes de producción y conservación de acuerdo a las condiciones biofísicas y socioeconómicos de cada región para la prescripción de las prácticas de manejo y las opciones que aseguren la conservación de los RFNM, y a la vez, el beneficio del sector social (FAO, 2010). Con el propósito de contribuir al respecto, la presente investigación documental tuvo como objetivo describir las investigaciones realizadas en los 35 años de existencia del Instituto Nacional de Investigaciones Forestales, Agrícolas y Pecuarias (INIFAP), referentes al aprovechamiento, manejo y conservación de los RFNM del norte de México. 


\section{Criterios de búsqueda y análisis de la información}

Se realizó una revisión temática del período 1985-2020 en diferentes repositorios científicos y revistas científicas disponibles en línea como ejemplo: SciELO (www.scielo.org/es), Redalyc (www.redalyc.org), Google Scholar (scholar.google.com), Revista Mexicana de Ciencias Forestales (https://cienciasforestales.inifap.gob.mx) y la Biblioteca digital del INIFAP (https://vun.inifap.gob.mx/BibliotecaWeb/_Content). Se consideraron todas las publicaciones científicas relacionadas a especies forestales no maderables de importancia económica del norte del país, desarrolladas por investigadores del INIFAP como primer autor; las cuales se ordenaron por taxón dentro de los programas de investigación del Instituto: Manejo Forestal Sustentable y Servicios Ambientales, Plantaciones y Sistemas Agroforestales, Recursos Genéticos Forestales no maderables, Biotecnología y Bioenergía.

\section{Área de estudio}

El área de estudio se acotó a las regiones semiáridas del norte de México donde se aprovechan los RFNM, que incluyen los estados de Baja California, Baja California Sur, Coahuila, Chihuahua, Durango, Nuevo León, San Luis Potosí, Sonora, Tamaulipas y Zacatecas.

\section{Manejo Forestal Sustentable y Servicios Ambientales}

En este programa de investigación se consideraron los temas: aprovechamiento sustentable de los recursos naturales, inventarios forestales, biometría de especies forestales no maderables en ecosistemas semiáridos del norte de México y modelación del potencial productivo, así como el mapeo de variables forestales. 


\section{Aprovechamiento sustentable de los recursos naturales}

En los diferentes tipos de matorral de las zonas áridas y semiáridas del norte del país se realiza el aprovechamiento de los RFNM; esta región ecológica comprende una superficie de 70.79 millones de hectáreas (Challenger y Soberón, 2008).

Las especies forestales no maderables presentan un aprovechamiento diverso, debido a las diferentes estructuras vegetales utilizables. Desde hace varios años se ha realizado de manera tradicional y sin sustento técnico. Por ello, en el INIFAP se realizan trabajos de investigación enfocados al aprovechamiento y manejo sustentable de estos recursos forestales de importancia comercial (Cuadro 1).

Cuadro 1. Especies no maderables aprovechables en el norte de México.

\begin{tabular}{|c|c|c|c|}
\hline Producto & Especie & Nombre Común & Referencia \\
\hline \multirow{5}{*}{ Fibra } & Agave lechuguilla Torr. & Lechuguilla & Berlanga et al. (1992) \\
\hline & Yucca carnerosana Trel. & Palma samandoca & Villavicencio (1993) \\
\hline & \multirow{3}{*}{ Nolina cespitifera Trel. } & \multirow{3}{*}{ Cortadillo } & Sáenz y Castillo (1992) \\
\hline & & & Castillo y Sáenz (1993) \\
\hline & & & Castillo et al. (2015) \\
\hline \multirow{2}{*}{ Cera } & \multirow{2}{*}{ Euphorbia antisyphilitica Zucc. } & \multirow{2}{*}{ Candelilla } & De la Garza y Berlanga (1993) \\
\hline & & & De la Garza et al. (1992) \\
\hline \multirow{4}{*}{ Bebida alcohólica (sotol) } & \multirow{4}{*}{ Dasylirion spp. } & \multirow{4}{*}{ Sotol } & Melgoza et al. (2003) \\
\hline & & & Vega et al. (2006) \\
\hline & & & Cano et al. $(2005,2011)$ \\
\hline & & & Sierra at al. (2008) \\
\hline Aguamiel & Agave salmiana Otto ex Salm-Dyck. & Maguey verde & Castillo y Cano (2006) \\
\hline Bebida alcohólica (mezcal) & Agave durangensis & Maguey cenizo & Rosales-Serna et al. (2020) \\
\hline Hojas & Lippia graveolens HBK & Orégano & Sáenz y Villavicencio (1993) \\
\hline \multirow{5}{*}{ Carbón } & \multirow{5}{*}{ Prosopis spp. } & \multirow{5}{*}{ Mezquite } & Villanueva et al. (2004) \\
\hline & & & Ríos et al. (2011) \\
\hline & & & Valenzuela-Núñez et al. (2011) \\
\hline & & & Ríos-Saucedo et al. (2012) \\
\hline & & & Valenzuela-Núñez et al. (2013) \\
\hline
\end{tabular}

Los diferentes productos extraídos de cada especie (Cuadro 1) son la materia prima derivada del aprovechamiento del recurso forestal, sin un proceso de transformación. En cuanto a investigaciones sobre el periodo de regeneración que tienen las diversas 
estructuras vegetales que se aprovechan, en el INIFAP se han realizado estudios sobre la época de aprovechamiento y turno técnico; es decir, el tiempo desde la extracción hasta la recuperación de la estructura vegetal de la planta (Cuadro 2).

Cuadro 2. Época de aprovechamiento y turno técnico para especies forestales no maderables en el norte de México.

\begin{tabular}{|c|c|c|c|c|}
\hline Especie & $\begin{array}{c}\text { Estructura } \\
\text { aprovechable }\end{array}$ & Época corte & $\begin{array}{l}\text { Turno corte } \\
\text { (meses) }\end{array}$ & Referencia \\
\hline Agave lechuguilla Torr. & Cogollo $^{1}(\geq 25 \mathrm{~cm}$ altura $)$ & Otoño-Invierno & 14 a 25 & $\begin{array}{l}\text { Berlanga et al. (1992) } \\
\text { Castillo et al. (2008) } \\
\text { Narcia et al. (2012) }\end{array}$ \\
\hline Yucca carnerosana (Trel.) McKelvey & Cogollo $^{1}(\geq 30 \mathrm{~cm}$ altura $)$ & Verano-Otoño & $12-18$ & $\begin{array}{c}\text { Villavicencio y Franco, } \\
\text { (1992) }\end{array}$ \\
\hline Euphorbia antisyphilitica Zucc. & $\begin{array}{l}\text { Tallos ( } \geq 30 \mathrm{~cm} \text { altura y } \\
\text { diámetro cobertura } \geq 25 \\
\mathrm{~cm} \text { ) }\end{array}$ & Otoño-Invierno & 36 a 60 & $\begin{array}{c}\text { De la Garza et al. (1992) } \\
\text { De la Garza y Berlanga } \\
(1993)\end{array}$ \\
\hline Nolina cespitifera Trel. & Hojas ( $\geq 40$ a $50 \mathrm{~cm}$ ) & Otoño-Invierno & 12 a $21 *$ & Sáenz y Castillo (1992) \\
\hline Agave salmiana Otto ex Salm-Dyck. & Hojas & Verano & 84 a 96 & Castillo y Cano (2006) \\
\hline Lippia graveolens HBK. & Hojas & Verano-Otoño** & 4 a 12 & Villavicencio et al. (2010b) \\
\hline
\end{tabular}

${ }^{1}$ Conjunto de hojas de la zona de crecimiento apical; * $\operatorname{Sin}$ afectar la zona de crecimiento apical; ${ }^{* *}$ Hasta dos cortes por año, en función de la precipitación.

Para E. antisyphilitica, por su forma de crecimiento colonial, se estableció un aprovechamiento en el que se establece la extracción de $50 \%$ de la colonia para asegurar la regeneración del recurso, mediante rotación de las áreas de aprovechamiento (De la Garza et al., 1992). En el caso de $A$. salmiana, se determinó un sistema de rotación de áreas de aprovechamiento en plantaciones, además del trasplante de hijuelos para replante (Castillo y Cano, 2006). Las especies forestales no maderables responden a la interacción genotipo-ambiente, lo que genera ecotipos diferenciados y restringidos a un hábitat específico, con diferentes características de crecimiento y producción; situación que debe considerarse en su aprovechamiento. 
Los resultados de los estudios incluidos en los cuadros 1 y 2 han servido de base para elaborar normas oficiales mexicanas referentes a las especificaciones técnicas para el aprovechamiento sustentable de los recursos forestales no maderables de zonas áridas y semiáridas. Además, se utilizan en la elaboración de los Estudios Técnicos Justificativos (ETJ) para el aprovechamiento de taxones forestales no maderables.

El conocimiento del desarrollo y comportamiento de los taxa aprovechables del semidesierto, así como las buenas prácticas de manejo contribuyen al manejo sustentable de las poblaciones naturales.

\section{Inventarios Forestales}

El objetivo de los inventarios forestales es proveer información de la cuantificación de los recursos forestales, a partir de muestreos en campo. Al respecto en el INIFAP se han aplicado técnicas de muestreo para la evaluación de los RFNM del norte del país (Cuadro 3).

Cuadro 3. Técnicas de muestreo para evaluar RFNM y ámbito de aplicación.

\begin{tabular}{|c|c|c|c|}
\hline Especie & $\begin{array}{c}\text { Tipo de } \\
\text { muestreo }\end{array}$ & $\begin{array}{l}\text { Entidad de } \\
\text { publicación }\end{array}$ & Referencia \\
\hline Agave lechuguilla Torr. & 1 & \multirow{3}{*}{$\begin{array}{c}\text { Coahuila, Nuevo } \\
\text { León, Chihuahua, } \\
\text { Durango, Zacatecas }\end{array}$} & Berlanga et al. (1992) \\
\hline Euphorbia antisyphilitica Zucc. & 1 & & $\begin{array}{c}\text { De la Garza et al. (1992) } \\
\text { De la Garza y Berlanga (1993) } \\
\text { Hernández et al. (2017) }\end{array}$ \\
\hline Dasylirion spp. & 3,4 & & $\begin{array}{l}\text { Cano et al. (2005) } \\
\text { Cano et al. (2011) }\end{array}$ \\
\hline Yucca carnerosana (Trel.) McKelvey & 2 & \multirow[b]{2}{*}{ Coahuila } & Villavicencio y Franco (1992) \\
\hline Nolina cespitifera Trel. & 2 & & $\begin{array}{c}\text { Sáenz y Castillo (1992) } \\
\text { Sáenz y Villavicencio (1993) }\end{array}$ \\
\hline Lippia graveolens HBK. & 1,2 & $\begin{array}{c}\text { Coahuila, Nuevo } \\
\text { León, Chihuahua, } \\
\text { Durango, } \\
\text { Tamaulipas, } \\
\text { Zacatecas }\end{array}$ & $\begin{array}{l}\text { Villavicencio et al. (2010b) } \\
\text { Villavicencio et al. (2018) }\end{array}$ \\
\hline
\end{tabular}

${ }^{1}$ Simple aleatorio, sitios de $100 \mathrm{~m}^{2}$; ${ }^{2}$ Sistemático, sitios de $100 \mathrm{~m}^{2}$, intensidad de muestreo de $0.5 \%$; ${ }^{3}$ Simple aleatorio, sitios de $1000 \mathrm{~m}^{2} ;{ }^{4}$ Sistemático, sitios de $1000 \mathrm{~m}^{2}$, intensidad de muestreo de $3 \%$. 
El muestreo sistemático es útil en rodales con distribución uniforme de especies como $Y$. carnerosana y $N$. cespitifera. En cuanto, al muestreo aleatorio estratificado es factible para evaluar poblaciones de A. lechuguilla, E. antisyphilitica, L. graveolens y Dasylirion spp. en áreas donde la distribución de los individuos es heterogénea. Estos muestreos son confiables para evaluar los taxones antes citados. Para L. graveolens, se realizó un análisis estructural de su población en su área de distribución del recuso, lo que evidenció la necesidad de establecer acciones de reforestación en las zonas deterioradas (Cuadro 1). Este tipo de investigaciones realizadas en el INIFAP contribuyen a la sustentabilidad de los RFNM, ya que en ellas se evalúa la productividad del ecosistema donde se desarrollan dichos recursos, y con ello se aseguran su aprovechamiento sustentable.

El uso de sistemas de muestreo apropiados para cada especie y condición de desarrollo, permite tener una cuantificación más precisa del recurso y optimizar los costos y tiempo en la elaboración y ejecución de los inventarios forestales.

\section{Biometría de especies forestales no maderables en ecosistemas semiáridos del norte de México}

La biometría es una herramienta para la estimación de las variables de la vegetación, se usa para cuantificar las existencias totales en un rodal. Al respecto, en el INIFAP se han desarrollado investigaciones que han derivado en el ajuste de 24 modelos matemáticos y la generación de cuatro tablas de producción para estimar variables de especies no maderables de ecosistemas semiáridos en el norte y noreste de México (Cuadro 4). Tablas que son de utilidad para la elaboración de planes de manejo sostenible y en toma de decisiones para el aprovechamiento de los RFNM de esas regiones. 
Cuadro 4. Investigaciones sobre modelos para estimar variables forestales de especies no maderables en el norte y noreste de México.

\begin{tabular}{|c|c|c|c|c|}
\hline Especie & Variable & $\begin{array}{l}\text { Área de } \\
\text { aplicación }\end{array}$ & Estructura & Referencia \\
\hline \multirow{4}{*}{ Agave lechuguilla Torr. } & Biomasa del cogollo & Coahuila & Tabla de producción & Berlanga et al. (1992) \\
\hline & & & $P S F=0.00040 D B C^{1.52498} A T C^{2.20623}$ & \multirow{3}{*}{ Velasco et al. (2009) } \\
\hline & Peso seco de fibra (PSF) & San Luis Potosí & $P S F=0.00078 D B C^{1.64385} A T C^{1.33236}$ & \\
\hline & & & $P S F=0.00038 D B C^{1.82763} A T C^{1.43348}$ & \\
\hline Yucca carnerosana (Trel.) McKelvey & Biomasa del cogollo & Coahuila & Tabla de producción & Villavicencio y Franco (1992) \\
\hline \multirow{4}{*}{ Euphorbia antisyphilitica Zucc. } & \multirow{4}{*}{ Peso verde total (PVT) } & \multirow{4}{*}{ Coahuila } & Tabla de producción & De la Garza y Berlanga (1993) \\
\hline & & & $P V T=5.125135 \operatorname{Exp}\left(\frac{-60.80586}{D P C}\right)$ & \multirow{3}{*}{ Hernández-Ramos et al. (2019) } \\
\hline & & & $P V T=0.004682(D P B)^{1.127425}(D P C)^{0.841933}$ & \\
\hline & & & $P V T=0.003614(D P B)^{0.48315}(D P C)^{1.268382}$ & \\
\hline \multirow{3}{*}{ Atriplex canescens (Pursh) Nutt. } & Peso seco de brotes (PSB) & \multirow{3}{*}{ Coahuila } & $P S B=0.30472(D P)^{1.61135}$ & \multirow{3}{*}{ Meza (1997) } \\
\hline & Peso seco de Ramas (PSR) & & $P S R=0.3405(D P)^{2.44254}$ & \\
\hline & Peso seco total (PST) & & $P S T=0.03745(D P)^{2.21993}$ & \\
\hline \multirow{5}{*}{ Prosopis spp. } & Volumen aprovechable & $\begin{array}{l}\text { Durango y } \\
\text { Coahuila }\end{array}$ & $V=0.0108+0.000057\left(D^{3} H\right)$ & Villanueva et al. (2004) \\
\hline & Volumen por tallo & \multirow{4}{*}{ Chihuahua } & $L n V=-10.58445+2.10597 \operatorname{Ln} D P B+0.90146 \operatorname{Ln} A T$ & \multirow{4}{*}{ Sosa et al. (2011) } \\
\hline & Volumen comercial total & & $\operatorname{LnV}=-10.55051+0.97614 \operatorname{Ln} A B+1.30718 \operatorname{Ln} A T$ & \\
\hline & Carbono aéreo por tallo (Ct) & & $L n C t=-3.13882+0.87661 \operatorname{Ln}\left(D B^{2} A T\right)$ & \\
\hline & $\begin{array}{l}\text { Carbono aéreo árbol } \\
\text { completo }(\mathrm{Cc})\end{array}$ & & $\operatorname{LnCc}=-2.76123+0.48339 \operatorname{Ln}\left(A B^{2} A T\right)$ & \\
\hline Dasylirion cedrosanum Trel. & Peso de piña (PP) & Coahuila & $P P=-15.529+1.118($ Dpi $)$ & Cano et al. (2005) \\
\hline Nolina cespitifera Trel. & Peso de hojas secas (PHS) & $\begin{array}{l}\text { Sur de } \\
\text { Coahuila }\end{array}$ & $L n P H S=\operatorname{Ln}(-1.143359+1.911099 \operatorname{Ln} D P B)$ & Castillo y Sáenz (2005) \\
\hline \multirow{4}{*}{ Lippia graveolens HBK } & Peso de hojas secas (PHS) & Coahuila & Tabla de producción & Villavicencio et al. (2010b) \\
\hline & Índice de área foliar (IAF) & \multirow{2}{*}{ Durango } & $I A F=0.1146+0.0101 L A P-0.00004 L A P^{2}$ & \multirow{2}{*}{ Villa-Castorena et al. (2011) } \\
\hline & Biomasa total planta (BTP) & & $B T P=-20.28+365.6 I A F$ & \\
\hline & Peso de hojas secas (PHS) & Coahuila & $P H S=0.00599(D P)^{1.935454}(A T)^{0.256803}$ & Villavicencio et al. (2018) \\
\hline Litsea parvifolia (Hemsl.) Mez & Peso de hojas secas (PHS) & Coahuila & $P H S=0.00147(D P)^{1.993821}(A T)^{0.492306}$ & $\begin{array}{c}\text { Villavicencio-Gutiérrez et al. } \\
(2020 \mathrm{c})\end{array}$ \\
\hline
\end{tabular}

$D P=$ Diámetro promedio $(\mathrm{cm}) ; D p i=$ Diámetro de piña; $D P B=$ Diámetro promedio de la base $(\mathrm{cm}) ; D P C=$ Diámetro promedio de cobertura $(\mathrm{cm}) ; D B C=$ Diámetro basal del cogollo $(\mathrm{cm}) ; A T=$ Altura total $(\mathrm{cm}) ; A B=$ Área basal del árbol; $D B=$ Diámetro basal del tallo; $A T C=$ Altura total del cogollo $(\mathrm{cm}) ; A M=$ Altura media $(\mathrm{cm}) ; V=$ Volumen $\left(\mathrm{m}^{3}\right) ; L A P=$ Lámina de agua aplicada $(\mathrm{cm}) ; \operatorname{Exp}=$ Exponencial;

$$
L n=\text { Logaritmo natural. }
$$




\section{Modelación del potencial productivo y mapeo de variables forestales}

En el manejo forestal es de suma importancia realizar estimaciones precisas de la cantidad de materia prima disponible para su aprovechamiento en un rodal forestal; para ello, en el INIFAP se realizan estudios enfocados al manejo forestal sustentable y a la generación de herramientas técnicas para la selección de sitios donde es factible el establecimiento de plantaciones forestales tanto comerciales (PFC), como de restauración (PFR) en el norte de México. En estos temas destacan la determinación de áreas potenciales para la implementación de PFC y áreas con potencial productivo para especies forestales no maderables (Cuadro 5); así como el mapeo de variables del rodal para la estimación de existencias totales en un inventario forestal.

Cuadro 5. Investigaciones sobre áreas potenciales para el establecimiento de PFC y potencial productivo por especie.

\begin{tabular}{|c|c|c|c|c|}
\hline Especie & Potencial & Área de aplicación & $\begin{array}{c}\text { Superficie potencial } \\
\text { (ha) }\end{array}$ & Referencia \\
\hline \multirow{2}{*}{ Nolina cespitifera Trel. } & PFC & Coahuila & $\begin{array}{l}\text { Alto: } 14128 \\
\text { Medio: } 66826\end{array}$ & Martínez y Castillo (2007) \\
\hline & Productivo & $\begin{array}{l}19 \text { estados del centro y norte } \\
\text { de México }\end{array}$ & 14138623 & Martínez et al. (2014) \\
\hline \multirow{6}{*}{ Dasylirion cedrosanum Trel. } & PFC & Coahuila & $\begin{array}{l}\text { Alto: } 2629969 \\
\text { Medio: } 4718910\end{array}$ & Cano y Martínez (2007) \\
\hline & \multirow{5}{*}{ Productivo } & Coahuila & 2202098 & Martínez et al. (2010) \\
\hline & & San Luis Potosí & 820702 & Beltrán et al. (2011) \\
\hline & & Nuevo León & 341386 & Martínez et al. (2011b) \\
\hline & & $\begin{array}{l}\text { Chihuahua, Coahuila, } \\
\text { Durango, Zacatecas }\end{array}$ & 6509324 & Martínez (2013) \\
\hline & & $\begin{array}{l}16 \text { estados del centro y norte } \\
\text { de México }\end{array}$ & 16673562 & Martínez et al. (2014) \\
\hline \multirow{7}{*}{ Agave lechuguilla Torr. } & \multirow{3}{*}{ PFC } & Coahuila & $\begin{array}{l}\text { Alto: } 83200 \\
\text { Medio: } 1194877\end{array}$ & Martínez et al. (2011a) \\
\hline & & Coahuila & 5159273 & Castillo-Quiroz et al. (2014a) \\
\hline & & Tamaulipas & 106272 & Castillo-Quiroz et al. (2014b) \\
\hline & \multirow{4}{*}{ Productivo } & Coahuila & 5446971 & Martínez et al. (2010) \\
\hline & & San Luis Potosí & 357596 & Beltrán et al. (2011) \\
\hline & & Nuevo León & 240382 & Martínez et al. (2011b) \\
\hline & & $\begin{array}{l}\text { Chihuahua, Coahuila, } \\
\text { Durango, Zacatecas }\end{array}$ & 8912445 & Martínez (2013) \\
\hline
\end{tabular}




\section{Villavicencio-Gutiérrez et al., Manejo forestal sustentable de los recursos...}

\begin{tabular}{|c|c|c|c|c|}
\hline & & $\begin{array}{l}16 \text { estados del centro y norte } \\
\text { de México }\end{array}$ & 17608351 & Martínez et al. (2014) \\
\hline \multirow{8}{*}{ Euphorbia antisyphilitica Zucc. } & \multirow{8}{*}{ Productivo } & Coahuila & 1828300 & Martínez et al. (2010) \\
\hline & & San Luis Potosí & 60970 & Beltrán et al. (2011) \\
\hline & & Nuevo León & 102598 & Martínez et al. (2011b) \\
\hline & & $\begin{array}{l}\text { Chihuahua, Coahuila, } \\
\text { Durango, Zacatecas }\end{array}$ & 8526336 & Martínez (2013) \\
\hline & & \multirow{3}{*}{$\begin{array}{l}20 \text { estados del centro y norte } \\
\text { de México }\end{array}$} & Alta: 7955139 & \multirow{3}{*}{ Zamora-Martínez et al. (2013) } \\
\hline & & & Media: 14512803 & \\
\hline & & & Baja: 8045010 & \\
\hline & & $\begin{array}{l}11 \text { estados del centro y norte } \\
\text { de México }\end{array}$ & 10520849 & Martínez et al. (2014) \\
\hline \multirow{5}{*}{ Agave salmiana Otto ex Salm-Dyck. } & \multirow{2}{*}{ PFC } & \multirow{2}{*}{ Zacatecas } & Alto: 1223887 & \multirow{2}{*}{ Medina et al. (2003) } \\
\hline & & & Medio: 743973 & \\
\hline & \multirow{3}{*}{ Productivo } & Coahuila & 5206366 & Martínez et al. (2010) \\
\hline & & San Luis Potosí & 2561217 & Beltrán et al. (2011) \\
\hline & & Nuevo León & 847337 & Martínez et al. (2011b) \\
\hline \multirow{2}{*}{ Agave tequilana Weber } & \multirow{2}{*}{ PFC } & \multirow{2}{*}{ Zacatecas } & Alto: 222434 & \multirow{2}{*}{ Medina et al. (2003) } \\
\hline & & & Medio: 442622 & \\
\hline Agave salmiana subp. crassispina & Productivo & Zacatecas & 61520 & Martínez (2013) \\
\hline \multirow{4}{*}{ Atriplex canescens (Pursh) Nutt } & \multirow{4}{*}{ Productivo } & Coahuila & 7411315 & Martínez et al. (2010) \\
\hline & & San Luis Potosí & 1604717 & Beltrán et al. (2011) \\
\hline & & Nuevo León & 1612598 & Martínez et al. (2011b) \\
\hline & & $\begin{array}{l}16 \text { estados del centro y norte } \\
\text { de México }\end{array}$ & 36201781 & Martínez et al. (2014) \\
\hline \multirow{4}{*}{ Parthenium argentatum A. Gray } & \multirow{4}{*}{ Productivo } & Coahuila & 5446971 & Martínez et al. (2010) \\
\hline & & San Luis Potosí & 2120049 & Beltrán et al. (2011) \\
\hline & & Nuevo León & 477517 & Martínez et al. (2011b) \\
\hline & & $\begin{array}{l}17 \text { estados del centro y norte } \\
\text { de México }\end{array}$ & 105621414 & Martínez et al. (2014) \\
\hline \multirow{4}{*}{ Dalea bicolor Humb. } & \multirow{4}{*}{ Productivo } & Coahuila & 1685163 & Martínez et al. (2010) \\
\hline & & San Luis Potosí & 1561026 & Beltrán et al. (2011) \\
\hline & & Nuevo León & 798400 & Martínez et al. (2011b) \\
\hline & & 25 estados de México & 24984153 & Martínez et al. (2014) \\
\hline \multirow{4}{*}{ Opuntia rastrera F.A.C. Weber } & \multirow{4}{*}{ Productivo } & Coahuila & 5953843 & Martínez et al. (2010) \\
\hline & & San Luis Potosí & 2467056 & Beltrán et al. (2011) \\
\hline & & Nuevo León & 2320610 & Martínez et al. (2011b) \\
\hline & & $\begin{array}{l}19 \text { estados del centro y norte } \\
\text { de México }\end{array}$ & 14470825 & Martínez et al. (2014) \\
\hline \multirow{4}{*}{ Lippia graveolens HBK. } & \multirow{4}{*}{ Productivo } & Coahuila & 907757 & Martínez et al. (2010) \\
\hline & & San Luis Potosí & 361435 & Beltrán et al. (2011) \\
\hline & & Nuevo León & 172994 & Martínez et al. (2011b) \\
\hline & & $\begin{array}{l}\text { Chihuahua, Coahuila, } \\
\text { Durango, Zacatecas }\end{array}$ & 6788321 & Martínez (2013) \\
\hline
\end{tabular}


En la determinación del potencial productivo o áreas para PFC, los autores (Cuadro 5) emplearon características del medio físico óptimas para el desarrollo de cada especie, mediante técnicas de Sistemas de Información Geográfica (SIG) para unificar dichos requerimientos agroclimáticos.

En cuanto a investigaciones sobre ordenamiento territorial, se ha propuesto para Coahuila (Martínez et al., 2010), Nuevo León (Martínez et al., 2011b) y San Luis Potosí (Beltrán et al., 2011) un ordenamiento territorial de las actividades productivas para el desarrollo social y económico de su población, a partir de las premisas de conservación, restauración y sustentabilidad de los recursos forestales no maderables.

\section{Plantaciones y Sistemas Agroforestales}

La demanda de materia prima de los RFNM se ha incrementado en los últimos años, la que se ha cubierto preferentemente de las poblaciones naturales; cuya producción está sujeta a las condiciones ambientales. El efecto de la sobreexplotación dificulta la recolecta del recurso, lo que conlleva a una inversión de más tiempo y esfuerzo para su realización, sin mayor remuneración (Castillo et al., 2015). Para mitigar la degradación de estos RFNM, en el INIFAP se hace investigación sobre el establecimiento de plantaciones forestales no maderables con fines de restauración o comerciales, para rehabilitar ecosistemas degradados, incrementar la densidad y materia prima, así como para mejorar la calidad del producto (Cuadro 6). 
Cuadro 6. Germoplasma forestal no maderable para el establecimiento de plantaciones en el norte de México.

\begin{tabular}{|c|c|c|}
\hline Especie & Tema & Autor \\
\hline Yucca carnerosana (Trel.) McKelvey & Material de propagación & Villavicencio (1993) \\
\hline Agave salmiana Otto ex Salm-Dyck. & $\begin{array}{l}\text { Selección de especies } \\
\text { Selección y manejo del material vegetativo }\end{array}$ & Castillo y Cano (2006) \\
\hline Agave durangensis Gentry & \multirow{2}{*}{ Fertilización química de plantaciones } & \multirow{2}{*}{ Rosales-Serna et al. (2020) } \\
\hline Dasylirion spp. & & \\
\hline \multirow{3}{*}{ Nolina cespitifera Trel. } & Pretratamientos químicos y biológicos para germinación & Castillo-Quiroz et al. (2018) \\
\hline & Calidad de planta & \multirow{2}{*}{ Sáenz et al. (2019) } \\
\hline & Producción y fertilización de planta en vivero & \\
\hline & Selección de ecotipos & \\
\hline Euphorbia antisyphilitica Zucc. & $\begin{array}{l}\text { Acondicionamiento, época y método de colecta del material de } \\
\text { plantación }\end{array}$ & De la Garza et al. (1992) \\
\hline \multirow{5}{*}{ Prosopis spp. } & Colecta y beneficio del germoplasma & \multirow{5}{*}{ Ramírez y Villanueva (1998) } \\
\hline & Tratamientos pregerminativos & \\
\hline & Calidad de planta & \\
\hline & Producción de planta en vivero & \\
\hline & Acondicionamiento de la planta & \\
\hline \multirow{4}{*}{ Lippia graveolens HBK. } & Calidad física y fisiológica de la semilla & \multirow{4}{*}{$\begin{array}{l}\text { Villa-Castorena (2011) } \\
\text { Cazares et al. (2010) }\end{array}$} \\
\hline & Esquema de producción de planta vivero e invernadero & \\
\hline & Nutrición, riego y densidad de plantación & \\
\hline & Aceites esenciales & \\
\hline
\end{tabular}

La tecnología generada en PFC demuestra que el monocultivo y domesticación es factible para las especies no maderables, y que bajo manejo se puede reducir el turno técnico de aprovechamiento. En el caso de A. lechuguilla, en condiciones naturales el turno varió de 24 a 25 meses (Narcia et al., 2012); mientras que, en PFC se reduce a 10 meses con un rendimiento de 220 y $560 \mathrm{~kg} \mathrm{ha}^{-1}$ (Castillo et al., 2008). Igualmente, en L. graveolens el turno de 10 a 12 meses en condiciones naturales, disminuye a cuatro meses en PFC con rendimientos de $3 \mathrm{t} \mathrm{ha}^{-1}$, a los tres años de su establecimiento (Villavicencio et al., 2010b). La implementación y manejo de plantaciones forestales comerciales con especies de las zonas áridas y semiáridas es una estrategia viable para la rehabilitación de los ecosistemas degradados y para cubrir el déficit de materia prima obtenida de poblaciones naturales (Cuadro 7). 
Cuadro 7. Investigaciones sobre el establecimiento y manejo de plantaciones forestales no maderables en el norte de México.

\begin{tabular}{|c|c|c|c|}
\hline Especie & Tema & $\begin{array}{c}\text { Densidad } \\
(\text { plantas ha-1) }\end{array}$ & Autor \\
\hline \multirow{2}{*}{ Agave lechuguilla Torr. } & Reconversión áreas productivas & 20000 & Berlanga et al. (1992) \\
\hline & \multirow[t]{2}{*}{ Establecimiento y manejo de PFC } & 20000 & Castillo et al. (2013) \\
\hline Euphorbia antisyphilitica Zucc. & & 20000 & De la Garza et al. (1992) \\
\hline Nolina-cespitifera Trel. & \multirow{2}{*}{$\begin{array}{l}\text { Reconversión áreas agrícolas } \\
\text { Establecimiento y manejo de PFC }\end{array}$} & 2500 & Castillo y Sáenz (2005) \\
\hline Prosopis spp. & & 1100 y 2500 & Ramírez y Villanueva (1998) \\
\hline
\end{tabular}

\section{Recursos Genéticos Forestales No Maderables}

Los recursos fitogenéticos para la alimentación y la agricultura (RFAA) se refieren a cualquier material de origen vegetal, sean plantas, semillas o partes vegetativas que contienen unidades funcionales de herencia con valor real o potencial para la alimentación y la agricultura (Gutiérrez et al., 2015). La accidentada orografía y la gran variedad de condiciones climáticas que existen en las zonas áridas y semiáridas del norte del país generan una gama de ambientes con diferentes tipos de vegetación, en donde la Familia Cactaceae tiene la mayor diversidad de géneros y especies, muchas de ellas endémicas y en estatus de riesgo según NOM-059-ECOL-2010 (Semarnat, 2010) y los Apéndices I y II de la Convención sobre el Comercio Internacional de Especies Amenazadas (CITES, 2019).

En el INIFAP, la conservación ex situ de recursos fitogenéticos de importancia se realiza en el Banco de Germoplasma de Cactáceas (BGC) in vivo, con taxones de los géneros y accesiones de la tribu Cacteae: Ariocarpus, Astrophytum, Aztekium, Coryphantha, Echinocactus, Epithelantha, Ferocactus, Geohintonia, Leuchtenbergia, Mammillaria, Mammilloydia, Pelecyphora, Stenocactus, Thelocactus y Turbinicarpus; así como de la tribu Pachycereeae: Cephalocereus, Echinocereus y Pachycereus. En dicho Banco se siguen los lineamientos de los RFAA del Sistema Nacional de Inspección y Certificación de Semillas (SNICS) (SNICS, 2018) y la Unión Internacional 
para la Protección de las Obtenciones Vegetales (UPOV, 2011) para constituir la base de datos del germoplasma nativo del Desierto Chihuahuense, de acuerdo al sistema de información Germocalli (Upadhyaya et al., 2008).

Al respecto, se han realizado estudios sobre distribución, en los que se evaluaron ocho subprovincias fisiográficas de la Sierra Madre Oriental, se elaboraron de mapas de riqueza de especies; se hizo la descripción taxonómica de los géneros Turbinicarpus (Villavicencio et al., 2006; Arredondo y Sotomayor, 2009; Villavicencio et al., 2010a; Arias et al., 2018), Mammillaria y Pelecyphora (Sotomayor et al., 2006); la caracterización morfológica de material nativo (Villavicencio et al., 2013a; Villavicencio et al., 2013b) para realizar una descripción sistemática de atributos cualitativos y cuantitativos de las entradas o accesiones de una misma especie o cultivo, tanto para diferenciarlas como para identificar genes o atributos específicos.

Además, se ha generado tecnología sobre regeneración y multiplicación, con resultados en la propagación por semilla de varios taxones, se han llevado a cabo pruebas física y fisiológica de semilla declarada; asimismo, se ha estudiado la propagación de diversos taxa no maderables en los que se evalúan sustratos, nutrición y fertirriego (Arredondo y Camacho, 1995; Arredondo, 2002; Flores et al., 2005; Vega et al., 2006; Villavicencio et al., 2019a), y la producción en invernadero de planta de ornato en contenedor (Villavicencio et al., 2020a).

Las acciones realizadas en el BGC contribuyen al manejo de los recursos fitogenéticos nativos, ya que en sus instalaciones se conserva el pool genético ex situ, que permite realizar acciones para promover su uso, potenciación, premejoramiento y constituye la materia prima para la conservación de germoplasma a corto y largo plazo. Esto, como una forma de conservación y uso sustentable de estos recursos fitogenéticos. En el BGC se han abordado las cuatro áreas estratégicas del Segundo Plan de Acción Mundial de la Organización de las Naciones Unidas para la Alimentación y la Agricultura (FAO, por sus siglas en inglés) sobre los RFAA, y se ha generado información para ocho de las 18 líneas prioritarias y en 10 indicadores. 
Las investigaciones se han enfocado en la región noreste de México; sin embrago, se requiere continuar con la recolección selectiva, el incremento del porcentaje de accesiones con duplicados de seguridad, así como con la regeneración y el aumento de las muestras ex situ. La información generada es fundamental para la sustentabilidad de estos recursos fitogenéticos que, por su valor cultural, económico o de relevancia para los ecosistemas requieren de su recuperación, protección, uso responsable y de una distribución justa y equitativa del beneficio que se obtiene como producto de su aprovechamiento sostenible.

\section{Biotecnología}

Para la conservación ex situ de recursos fitogenéticos de la Familia Cactaceae, en el INIFAP se tiene un banco de tejidos in vitro para preservar germoplasma de especies en estatus de riesgo y de interés ornamental; en cuya conformación se han aplicado herramientas biotecnológicas, mediante el cultivo de tejidos vegetales. Al respecto, se tienen resultados en la germinación in vitro, inducción de brotes a partir de yemas axilares en la multiplicación, selección del tipo y concentración de fitohormonas en la inducción de brotes, así como manejo de las vitroplantas en la aclimatación (Villavicencio et al., 1999).

Se han logrado establecer protocolos de micropropagación para Astrophytum myriostigma Lem. (Villavicencio et al., 2009), Turbinicarpus knuthianus (Boed.) John \& Riha (Villavicencio et al., 2011; Villavicencio et al., 2012a), Epithelantha micromeris (Engelm.) F.A.C. Weber ex Britton. \& Rose (Villavicencio et al., 2012b; Villavicencio et al., 2012c), Echinocereus poselgeri Lem. (Villavicencio et al., 2020b), Turbinicarpus viereckii subsp. major Glass \& R.A. Foster y Mammillaria plumosa F.A.C. La tecnología producida se ha transferido a productores del Comité Mexicano Sistema-Producto Flores y Ornamentales (CMSPFO).

Para la industria del sotol, se generó el protocolo de micropropagación para $D$. cedrosanum, especie empleada para producción de esta bebida alcohólica con 
denominación de origen (Villavicencio et al., 2007); esta tecnología se transfirió a la empresa Tequila Sauza S. A. de C. V., para lo cual se consideró su experiencia en la propagación industrial de plantas de agave.

\section{Bioenergía}

Actualmente, la producción de biocombustibles de segunda generación (2G) a partir de biomasa lignocelulósica de plantas silvestres de zonas semiáridas se ha convertido en un tema de interés para el sector energético (Carmona et al., 2017); no obstante, es un campo en reciente desarrollo. Dentro de las especies nativas de las zonas semiáridas con potencial y con más estudios realizados para la producción de etanol destaca: $A$. lechuguilla, cuya biomasa no compite con la producción de alimentos, en comparación con los biocombustibles de primera generación (1G) que se obtienen de materias primas de cultivos agrícolas como el maíz y la caña de azúcar (Serna et al., 2011).

A. lechuguilla posee un gran potencial por su amplia superficie de distribución en el país (17.5 millones de hectáreas) (Martínez et al., 2014), la existencia de una Norma Oficial Mexicana para su aprovechamiento (Semarnat, 2003); además, es una planta con una alta digestibilidad enzimática, por lo que es importante para el sector forestal. Al respecto, los investigadores del INIFAP han colaborado con un grupo interdisciplinario de la Universidad Autónoma de Coahuila (UAC) en varios estudios, cuyos resultados se resumen en el Cuadro 8.

Cuadro 8. Investigaciones sobre bioenergía para la obtención de etanol a partir de la biomasa de Agave lechuguilla Torr. en Coahuila, México.

\begin{tabular}{lc}
\hline \multicolumn{1}{c}{ Tema } & \multicolumn{1}{c}{ Autores } \\
\hline $\begin{array}{l}\text { Determinación de área potenciales para plantaciones de A. lechuguilla para } \\
\text { la producción de etanol } \\
\text { Potencial del } A \text {. lechuguilla como cultivo energético }\end{array}$ & Castillo et al. (2014b) \\
Propiedades químicas, estructurales y funcionales de A. lechuguilla & Morales et al. (2014) \\
Pretratamiento fúngico de la biomasa de A. lechuguilla & Carmona et al. (2017) \\
\hline
\end{tabular}


En la actualidad, $A$. lechuguilla es la especie más estudiada, principalmente, para la producción de etanol; sin embargo, se requiere realizar investigaciones de esta materia prima para la obtención de biocombustibles líquidos (butanol) y gaseosos (hidrógeno) y otras fuentes alternas como el biogás; por otro lado, en las zonas áridas existen otras especies nativas que al igual que $A$. lechuguilla son una fuente potencial para la producción de biocombustibles.

\section{Conclusiones}

El mayor número de contribuciones desarrolladas por investigadores del INIFAP en los últimos 35 años sobre especies no maderables del semidesierto en México, se concentra en los temas de ordenación y potencial productivo, biometría, establecimiento y manejo de plantaciones y en recursos fitogenéticos de especies en estatus de riesgo; en menor proporción, pero no menos importantes, son los temas sobre inventarios forestales y prácticas para el aprovechamiento, así como investigaciones en biocombustibles. En Coahuila es donde más se ha trabajado, lo que coincide con su importancia como el mayor generador de productos no maderables.

Es relevante señalar que todos los productos generados en el INIFAP han tenido un gran impacto en la sustentabilidad de los recursos naturales del semidesierto, y han contribuido de manera sobresaliente a mejorar las actividades productivas de las comunidades rurales, mismas que dependen del aprovechamiento de estas especies forestales.

Las aportaciones documentadas en la presente revisión pueden utilizarse para llevar a cabo programas de apoyo a comunidades de zonas marginadas para contribuir a mejorar su situación económica, así como a restaurar zonas ecológicas perturbadas, lo cual coadyuvará a proporcionar mayor protección a los ecosistemas naturales de las zonas áridas y semiáridas de México. 


\section{Conflicto de intereses}

Los autores declaran no tener conflicto de intereses.

\section{Contribución por autor}

Eulalia Edith Villavicencio-Gutiérrez, Antonio Cano-Pineda, David Castillo-Quiroz y Adrián Hernández-Ramos: compilación, redacción y revisión del documento; Oscar Ulises Martínez Burciaga: aportaciones de trabajos científicos significativos al documento.

\section{Referencias}

Arias M., S., E. E. Villavicencio G. y M. A. Carranza P. 2018. Biznagas y Nopales (Cactaceae). In: Comisión Nacional para el Conocimiento y Uso de la Biodiversidad (CONABIO) y Gobierno del Estado de Coahuila de Zaragoza (Eds.) La biodiversidad en Coahuila. Estudio de Estado Vol. II. CONABIO/Gobierno del Estado de Coahuila de Zaragoza. Saltillo, Coah., México. pp. 71-182. www.sema.gob.mx/SRN-CONSERESTUDIO-V2.php (8 de noviembre de 2020).

Arredondo G., A. y F. Camacho M. 1995. Germinación de Astrophytum myriostigma (Lemaire) en relación con la procedencia de las semillas y la temperatura de incubación. Cactáceas y Suculentas Mexicanas 40(2): 34-38.

web.ecologia.unam.mx/cactsucmex/csm1995_40_1.pdf (10 de septiembre de 2020).

Arredondo G., A. 2002. Propagación y mantenimiento de cactáceas. CIRNE-INIFAP, Campo Experimental San Luis. Folleto técnico No. 21. Soledad de Graciano Sánchez, SLP., México. 28 p. https://cultivandoflores.com/wpcontent/uploads/2020/06/Gu\%C3\%ADa-de-Propagaci\%C3\%B3n-y-Mantenimientode-Cactaceas-PDF-CultivandoFlores.Com_.pdf (12 de julio de 2021). 
Arredondo G., A. y J. M. Sotomayor. 2009. Cactáceas en categoría de riesgo del Estado de San Luis Potosí. CIRNE-INIFAP, Campo Experimental San Luis. Soledad de Graciano Sánchez, SLP., México. Publicación Especial No. 1. 114 p.

www.inifapcirne.gob.mx/Biblioteca/Publicaciones/811.pdf (2 septiembre de 2020).

Beltrán L., S., O. U. Martínez B., G. Medina G., C. Loredo O. y R. Gutiérrez L. 2011. Opciones de ordenamiento productivo de las regiones árida y semiárida del estado de San Luis Potosí. CIRNE-INIFAP, Campo Experimental San Luis. San Luis Potosí, SLP., México. Folleto técnico No. 43. 137 p.

www.inifapcirne.gob.mx/Biblioteca/Publicaciones/.pdf (2 de enero de 2021).

Berlanga R., C. A., L. A. González L. y H. Franco L. 1992. Metodología para la evaluación de lechuguilla en condiciones naturales. CIRNE-INIFAP, Campo Experimental Saltillo. Sitio Experimental La Sauceda. Saltillo, Coah., México. Folleto técnico No. 1. 22 p. www.inifapcirne.gob.mx/Biblioteca/Publicaciones/.pdf (2 de enero de 2021).

Cano P., A., C. A. Berlanga R., D. Castillo Q., O. U. Martínez B. y A. Zárate L. 2005. Análisis dimensional y tablas de producción de sotol (Dasylirion cedrosanum Trel.) para el estado de Coahuila. CIRNE-INIFAP, Campo Experimental Saltillo. Saltillo, Coah., México. Folleto técnico No. 18. 24 p. http://www.inifapcirne.gob.mx/Biblioteca/Publicaciones/182.pdf (2 de enero de 2021).

Cano P., A. y O. U. Martínez B. 2007. Determinación de áreas potenciales para el establecimiento de plantaciones de sotol (Dasylirion cedrosanum Trel.) en el estado de Coahuila. CIRNE-INIFAP, Campo Experimental Saltillo. Saltillo, Coah., México. Folleto técnico No. 31.28 p. www.inifapcirne.gob.mx/Biblioteca/Publicaciones/183.pdf (10 de septiembre de 2020). 
Cano P., A., O. U. Martínez B., C. A. Berlanga R., E. E. Villavicencio G. y D. Castillo Q. 2011. Guía para la evaluación de existencias de sotol (Dasylirion cedrosanum Trel.) en poblaciones naturales del Estado de Coahuila. CIRNE-INIFAP, Campo Experimental Saltillo. Saltillo, Coah., México. Folleto técnico No. 43. 29 p. www.inifapcirne.gob.mx/Biblioteca/Publicaciones/887.pdf (8 de noviembre de 2020). Carmona J., E. T. K. Morales-Martínez, M. Solange I., D. Castillo-Quiroz y L. RíosGonzález. 2017. Propiedades químicas, estructurales y funcionales de la lechuguilla (Agave lechuguilla Torr.). Revista Mexicana de Ciencias Forestales 8(42): 100-122. Doi: $10.29298 /$ rmcf.v8i42.21.

Castillo Q., D. y J. T. Sáenz R. 1993. Aspectos ecológicos del cortadillo Nolina sp. en el sur de Saltillo, General de Cepeda y Parras de la Fuente, Coahuila. CIRNE-INIFAP, Campo Experimental Saltillo. Sitio Experimental La Sauceda. Saltillo, Coah., México. Folleto técnico No. 4. 17 p. www.inifapcirne.gob.mx/Biblioteca/Publicaciones/pdf (8 de noviembre de 2020).

Castillo Q., D. y J. T. Sáenz R. 2005. Tarifa de rendimiento de cortadillo (Nolina cespitifera Trel.) para el sur de Coahuila. CIRNE-INIFAP, Campo Experimental Saltillo. Saltillo, Coah., México. Folleto técnico Núm. 19. 23 p.

www.inifapcirne.gob.mx/Biblioteca/Publicaciones/195.pdf (3 de octubre de 2020).

Castillo Q., D. y A. Cano P. 2006. Establecimiento y manejo de plantaciones comerciales de maguey para la extracción de aguamiel en el sureste de Coahuila. CIRNE-INIFAP, Campo Experimental Saltillo. Saltillo, Coah., México. Folleto técnico Núm. 23. 18 p. www.inifapcirne.gob.mx/Biblioteca/Publicaciones/199.pdf (10 de septiembre de 2020). 
Castillo Q., D., C. A. Berlanga R., M. Pando M. y A. Cano P. 2008. Regeneración del cogollo de Agave lechuguilla Torr. de cinco procedencias bajo cultivo. Revista Mexicana de Ciencias Forestales 33(103): 27-40.

http://cienciasforestales.inifap.gob.mx/editorial/index.php/forestales/article/view/739 (11 de octubre de 2020).

Castillo Q., D., J. T. Sáenz R., M. Narcia V. y J. A. Vázquez R. 2013. Propiedades físicomecánicas de la fibra de Agave lechuguilla Torr. de cinco procedencias bajo plantaciones. Revista Mexicana de Ciencias Forestales 4(19): 78-91. Doi: 10.29298/rmcf.v4i19.380.

Castillo-Quiroz, D., O. U. Martínez-Burciaga, D. Y. Ávila-Flores, F. Castillo-Reyes and J. D. Sánchez-Chaparro. 2014a. Identification of potential areas for establishment of plantations of Agave lechuguilla Torr. in Coahuila, México. Open Journal of Forestry 4: 520-526. Doi: 10.4236/ojf.2014.45056.

Castillo-Quiroz, D., O. U. Martínez-Burciaga, L. J. Ríos-González, J. A. Rodríguez-de la Garza, T. K. Morales-Martínez, F. Castillo-Reyes y D. Y. Ávila-Flores. 2014b. Determinación de área potenciales para plantaciones de Agave lechuguilla Torr. para la producción de etanol. Revista Acta Química Mexicana 3(12): 3-11. www.actaquimicamexicana.uadec. $m x / ? p=416$ (13 de septiembre de 2020).

Castillo Q., D., D. Y. Ávila F., F. Castillo R., A. Antonio B. y O. U. Martínez B. 2015. Nolina cespitifera Trel. Recurso forestal no maderable de importancia económica y social del noroeste de México. Interciencia 40(9): 611-617. www.redalyc.org/pdf/339/33940998005.pdf (22 de octubre de 2020).

Castillo-Quiroz, D., A. Bautista-Antonio, D. Y. Ávila-Flores, J. T. Sáenz R. y F. Castillo-Reyes. 2018. Tratamientos químicos y biológicos para estimular la germinación en semillas de Nolina cespitifera Trel. Polibotánica (45): 151-160. Doi: $10.18387 /$ polibotanica.45.11. 
Carmona J., E. T. K. Morales-Martínez, M. Solange I., D. Castillo-Quiroz y L. RíosGonzález. 2017. Propiedades químicas, estructurales y funcionales de la lechuguilla (Agave lechuguilla Torr.). Revista Mexicana de Ciencias Forestales 8(42): 100-122. Doi: $10.29298 /$ rmcf.v8i42.21.

Cazares A., N. P., E. E. Villavicencio G., J. Verde S., V. Pecina Q. y I. H. Alameyda L. 2010. Caracterización Molecular y Producción de Aceites Esenciales en Diferentes Genotipos de Orégano (Lippia sp.). Revista Mexicana de Ciencias Forestales 1(1): 85-94. Doi: 10.29298/rmcf.v1i1.656.

Challenger, A. y J. Soberón. 2008. Los ecosistemas terrestres. In: Alonso C., A., S. Anta, J. Carabias, R. Dirzo, E. Ezcurra, R. González, G. Halffter, J. LlorenteBousquets, I. March, J. de la Maza, A. Mohar, I. Pisanty, J. Sarukhán y J. Soberón. (comps.). Capital natural de México, vol. I. Comisión Nacional para el Conocimiento de la Biodiversidad. México, D.F., México. pp. 87-108. http://www2.biodiversidad.gob.mx/pais/pdf/CapNatMex/Vol\%20I/I00_PrefacioGuia.pdf (5 de agosto de 2021).

Convención sobre el comercio internacional de especies amenazadas (CITES). 2019. Apéndices I, II and III. CITES. Ginebra, Suiza. 80 p. https://cites.org/sites/default/files/esp/app/2019/S-Appendices-2019-11-26.pdf (2 de diciembre de 2020).

De la Garza P., F. E., C. A. Berlanga R. y F. J. Tovar V. 1992. Guía para el establecimiento y manejo de plantaciones de candelilla. CIRNE-INIFAP, Campo Experimental La Sauceda. Saltillo, Coah., México. Folleto técnico No. 2. 14 p. www.inifapcirne.gob.mx/Biblioteca/Publicaciones/.pdf (9 de julio de 2021).

De la Garza P., F. E. y C. A. Berlanga R. 1993. Metodología para la evaluación y manejo de Candelilla en condiciones naturales. CIRNE-INIFAP, Campo Experimental La Sauceda. Saltillo, Coah., México. Folleto técnico No. 5. 46 p. www.inifapcirne.gob.mx/Biblioteca/Publicaciones/.pdf (8 de enero de 2021). 
Organización de las Naciones Unidas para la Alimentación y la Agricultura (FAO). 2010. El Segundo Informe sobre el estado de los Recursos Fitogenéticos para la Alimentación y la Agricultura en el Mundo. Comisión de recursos genéticos para la alimentación y la agricultura. Roma, Italia. 28 p.

www.fao.org/3/i1500s/i1500s00.pdf (16 de septiembre de 2020).

Flores, J., A. Arredondo G. and E. Jurado. 2005. Comparative Seed Germination in Species of Turbinicarpus: an Endangered Cacti Genus. Natural Areas Journal 25(2): 183-187. www.researchgate.net/publication/236323883 (6 de enero de 2021).

Gutiérrez, B., R. Ipinza y S. Barros. 2015. Conservación de Recursos Genéticos Forestales, Principios y Prácticas. Instituto Forestal. Santiago, Chile. 320 p. www.researchgate.net/publication/284188875 (2 de octubre de 2020).

Hernández R., A., A. Cano P., C. Flores L., J. Hernández R., J. J. García M. y X. García C. 2017. Comparación de los estimadores de dos métodos de muestreo en poblaciones naturales de candelilla. Revista Mitigación del Daño Ambiental Agroalimentario y Forestal de México 3(3): 75-84. https://docplayer.es/89890820Revista-mitigacion-del-dano-ambiental-agroalimentario-y-forestal-de-mexico.html (4 de diciembre de 2020).

Hernández-Ramos, A., A. Cano-Pineda, C. Flores-López, J. Hernández-Ramos, X. García-Cuevas, M. Martínez-Salvador y L. Martínez-Ángel. 2019. Modelos para estimar biomasa de Euphorbia antisyphilitica Zucc. en seis municipios de Coahuila. Madera y Bosques 25(2): e2521806. Doi: 10.21829/myb.2019.2521806.

Instituto Nacional de Estadística y Geografía (Inegi). 2020. Cuéntame. www.cuentame.inegi.org.mx/. (18 de enero de 2020). 
Martínez B., O. U. y D. Castillo Q. 2007. Identificación de sitios para plantaciones de cortadillo Nolina cespitifera Trel. en el sureste de Coahuila. CIRNE-INIFAP, Campo Experimental Saltillo. Saltillo, Coah., México. Folleto técnico No. 34. 24 p. www.inifapcirne.gob.mx/Biblioteca/Publicaciones/220.pdf (11 de enero de 2021).

Martínez B., O. U., G. Medina G., C. Loredo O., M. Espinosa R., M. M. Silva S., H. Fuente S., R. Gutiérrez L., S. Beltrán L. y F. Moreno S. 2010. Propuesta de ordenamiento productivo de las regiones áridas y semiáridas del estado de Coahuila. CIRNE-INIFAP, Campo Experimental Saltillo. Saltillo, Coah., México. Publicación especial No. 15. 96 p.

www.inifapcirne.gob.mx/Biblioteca/Publicaciones/848.pdf (8 de enero de 2021).

Martínez B., O. U., D. Castillo Q. y O. Mares A. 2011a. Caracterización y selección de sitios para plantaciones de lechuguilla (Agave lechuguilla Torr.) en el estado de Coahuila. CIRNE-INIFAP, Campo Experimental Saltillo. Saltillo, Coah., México. Folleto técnico No. 47. 26 p. www.inifapcirne.gob.mx/Biblioteca/Publicaciones/886.pdf (5 de febrero de 2021). Martínez B., O. U., H. Fuente S. y G. Medina G. 2011b. Ordenamiento del uso de suelo para producción de especies no maderables de uso múltiple en el estado de Nuevo León. CIRNE-INIFAP, Campo Experimental Saltillo. Saltillo, Coah., México. Folleto técnico No. 45.30 p.

www.inifapcirne.gob.mx/Biblioteca/Publicaciones/884.pdf (8 de febrero de 2021).

Martínez S., M. 2013. Ecología y usos de especies forestales de interés comercial de las zonas áridas de México. CIRNO-INIFAP, Sitio Experimental La Campana. Cd. Aldama, Chih., México. Folleto técnico No. 5. 226 p. https://docplayer.es/70889081-Ecologia-y-usos-de-especies-forestales-de-interescomercial-de-las-zonas-aridas-de-mexico-martin-martinez-salvador.html (15 de febrero de 2021). 
Martínez B., O. U., A. González H., F. Moreno S., J. A. Ruíz C. y K. Trinidad L. 2014. Distribución potencial de especies no maderables de zonas áridas bajo diferentes escenarios climáticos en México. CIRNE-INIFAP, Campo Experimental Saltillo. Saltillo, Coah., México. Folleto técnico No. MX-0-310699-52-03-15-09-62. 44 p. www.inifapcirne.gob.mx/Biblioteca/Publicaciones/1011.pdf (9 de febrero de 2021).

Medina G., G., B. Cabañas C., J. A. Ruiz C., J. Madero T., S. Rubio D., A. Rumayor R., M. Luna F., C. Gallegos V., R. Gutiérrez V. y A. G. Bravo L. 2003. Potencial productivo de especies agrícolas en el estado de Zacatecas. CIRNOC-INIFAP, Campo Experimental Zacatecas. Zacatecas, Zac., México. Folleto técnico No. 2. 166 p. www.inifap-nortecentro.gob.mx/biblioteca_virtual_usuario.php (11 de febrero de 2021).

Melgoza C., A. y J. Sierra T. 2003. Contribución al conocimiento y distribución de las especies de Dasylirion spp. (sotol) en Chihuahua, México. Revista Mexicana de Ciencias Forestales 28(93): 25-40. https://cienciasforestales.inifap.gob.mx/editorial/index.php/forestales/article/view/888 (1 de julio de 2021).

Meza S., R. 1997. Ecuaciones para estimar la fitomasa de Atriplex canescens. Revista Ciencia Forestal en México 22(81): 27-40. https://cienciasforestales.inifap.gob.mx/index.php/busqueda (10 de enero de 2021). Morales M., T. K., J. Ríos G. L., J. A. Rodríguez de la G., Y. Garza G. y D. Castillo Q. 2014. Potencial del Agave lechuguilla como cultivo energético. Revista Cienciacierta 10: 13-15. www.cienciacierta.uadec.mx/2014/12/12/potencial-del-agavelechuguilla-como-cultivo-bioenergetico-en-mexico/ (8 de agosto de 2020).

Narcia V., M., D. Castillo Q., J. A. Vázquez R. y C. A. Berlanga R. 2012. Turno técnico de la lechuguilla (Agave lechuguilla Torr.) en el noreste de México. Revista Mexicana de Ciencias Forestales 3(9): 81-88. Doi: 10.29298/rmcf.v3i9.531. 
Ramírez G., J. A. y J. Villanueva D. 1998. Selección y Manejo de material reproductivo de mezquite (Prosopis spp). CIRNE-INIFAP, Campo Experimental Palma de la Cruz. San Luis Potosí, S.L.P., México. Folleto técnico No. 9. 20 p. www.inifapcirne.gob.mx/Biblioteca/Publicaciones/763.pdf (4 de noviembre de 2020).

Reyna-Martínez, R., T. K. Martínez-Morales, D. Castillo-Quiroz, J. C. ContrerasEsquivel y L. J. Ríos-González. 2019. Pretratamiento fúngico de biomasa de Agave lechuguilla Torr. Revista Mexicana de Ciencias Forestales 10(51): 86-106. Doi: $10.29298 /$ rmcf.v10i51.336.

Ríos S., J., R. Trucíos C., L. M. Valenzuela N., G. Sosa P. y R. Rosales S. 2011. Importancia de las poblaciones de mezquite en el norte-centro de México. Campo Experimental Valle del Guadiana. CIRNOC-INIFAP. Gómez Palacio, Dgo., México. Libro Técnico No. 8. 218 p. www.researchgate.net/publication/304749878 (1 de julio de 2021).

Ríos-Saucedo, J. C., R. Rosales-Serna, J. L. García-Rodríguez, R. Tracios-Caciano y L. M. Valenzuela-Núñez. 2012. Diagnóstico de reforestaciones de mezquite y métodos para incrementar su eficiencia en Durango, México. Revista Forestal Baracoa 31(2): 35-40. http://www.actaf.co.cu/revistas/rev_forestal/Baracoa-20122/FAO2\%202012/DIAGN\%C3\%93STICO\%20DE\%20REFORESTACIONES\%20DE\%20 MEZQUITE.pdf (1 de agosto de 2021).

Rosales-Serna, R., J. C. Ríos-Saucedo, S. Rosales-Mata, S. Santana-Espinoza y P. A. Domínguez-Martínez. 2020. Fertilización química y crecimiento de maguey cenizo y sotol en plantaciones comerciales establecidas en Durango. Ciencia e Innovación 3(1): 43-52. www.researchgate.net/publication/342393173 (3 de julio de 2021).

Sáenz R., J. T. y D. Castillo Q. 1992. Guía para la evaluación de cortadillo en el estado de Coahuila. CIRNE-INIFAP, Campo Experimental La Sauceda. Saltillo, Coah., México. Folleto técnico No. 3. 13 p. www.inifapcirne.gob.mx/Biblioteca/Publicaciones/ (10 de octubre de 2020). 
Sáenz R., J. T. y E. E. Villavicencio G. 1993. Guía para la evaluación de orégano en el estado de Coahuila. CIRNE-INIFAP, Campo Experimental La Sauceda. Saltillo, Coah., México. Folleto técnico No. 6. 16 p.

www.inifapcirne.gob.mx/Biblioteca/Publicaciones/ (20 de octubre de 2020).

Sáenz R., J. T., D. Castillo Q., F. Castillo R., H. J. Muñoz-Flores y D. Y. Ávila-Flores. 2019. Determinación de la calidad de planta en cortadillo (Nolina cespitifera Trel.) con fertilización química y biológica en vivero. Revista BioCiencias 6: e547. Doi: 10.15741/revbio.06.e547.

Secretaria del Medio Ambiente y Recursos Naturales (Semarnat). 2003. Norma Oficial Mexicana NOM-008-SEMARNAT-1996. Procedimientos, criterios y especificaciones para realizar aprovechamiento, transporte y almacenamiento de cogollos. Diario Oficial de la Federación. 24 de junio de 1996. México, D.F., México. www.profepa.gob.mx/innovaportal/file/3310/1/nom-008semarnat -1996.pdf (2 de septiembre de 2020).

Secretaria del Medio Ambiente y Recursos Naturales (Semarnat). 2010. Norma Oficial Mexicana NOM-059-ECOL-2010. Protección ambiental-Especies nativas de México de flora y fauna silvestres-Categorías de riesgo y especificaciones para su inclusión, exclusión o cambio. Lista de especies en riesgo. Diario Oficial de la Federación. 30 de diciembre de 2010. México, D.F., México. 130 p. https://dof.gob.mx (5 de diciembre de 2020).

Secretaria del Medio Ambiente y Recursos Naturales (Semarnat). 2020. Anuario estadístico de la producción forestal 2017. Dirección General de Gestión Forestal y de Suelos. México, CDMX., México. 284 p. http://dsiappsdev.semarnat.gob.mx/datos/portal/publicaciones/2020/2017.pdf (16 de marzo de 2021). 
Serna F., L. Barrera y H. Montiel. 2011. Impacto Social y Económico en el Uso de Biocombustibles. Journal of Technololgy Managagement \& Innovation (6): 100-114. Doi: $10.4067 /$ S0718-27242011000100009.

Servicio Nacional de Inspección y Certificación de Semillas (SNICS) 2018. Catálogo Nacional de Variedades Vegetales. 3er. Trimestre 2018. SAGARPA-SNICS. México, CDMX., México. 49 p. www.gob.mx/snics (20 de octubre de 2020).

Sierra T., J. S., C. R. Lara M., R. Carrillo R., A. Mendoza C., C. Morales N. y M. H. Royo M. 2008. Los sotoles (Dasylirion spp.) de Chihuahua. CIRNOC-INIFAP, Sitio Experimental La Campana-Madera. Aldama, Chih., México. Folleto técnico No. 20. 58 p. https://docplayer.es/41619410-Los-sotoles-dasylirion-spp-de-chihuahua.html (12 de julio de 2021).

Sosa P., G., J. C. Ríos S. N. Chávez S., J. A. Sígala R. y D. Albarrán A. 2011. Modelos para la estimación del volumen y carbono del mezquite (Prosopis spp) en el estado de Chihuahua. CIRNOC-INIFAP, Sitio Experimental La Campana-Madera. Aldama, Chih., México. Folleto técnico No. 38. 39 p.

www.researchgate.net/publication/283315604_Modelos_para_la_estimacion_del_vo lumen_de_carbono_del_mezquite_Prosopis_spp_en_el_Estado_de_Chihuahua (15 de marzo de 2021).

Sotomayor J., M., A. Arredondo G., F. R. Sánchez B. y M. Martínez M. 2006. Pelecyophora strobiliformis (Wedermann) Fric \& Schelle ex Kreuzinger (Cactaceae): a new locality in San Luis Potosí, México. Bradleya (24): 112-114. https://bioone.org/journals/bradleya/volume-2006/issue-24 (4 de febrero de 2021).

Unión Internacional Para la Protección de las Obtenciones Vegetales (UPOV). 2011. Directrices de examen. Documento TC/47/2. Ginebra, Suiza. 11 p. www.upov.int/edocs/mdocs/upov/es/c_47/c_47_2.pdf (4 de febrero de 2021). 
Upadhyaya, H. D., C.L.L. Gowda and D. V. S. S. R. Sastry. 2008. Plant genetic resources management: collection, characterization, conservation and utilization. Journal of SAT Agricultural Research 6:1-16.

Valenzuela-Núñez, L. M., R. Trucíos-Caciano, J. C. Ríos-Saucedo, A. Flores H. y J. L. González-Barrios. 2011. Caracterización dasométrica y delimitación de rodales de mezquite (Prosopis sp) en el estado de Coahuila. Chapingo Serie Ciencias Forestales y del Ambiente 17: 87-96. Doi: 10.5154/r.rchscfa. 2010.09.067.

Valenzuela-Núñez, L. M., M. Rivera-González, R. Trucíos-Caciano y J. C. RíosSaucedo. 2013. Características ecológicas y dasométricas de dos comunidades con mezquite (Prosopis laevigata [Humb. et Bonpl. ex Willd] M. C. Johnston) en el estado de Durango. Tecnociencia Cihuahua 7(1): 32-38.

http://tecnociencia.uach.mx/numeros/v7n1/Data/Caracteristicas_ecologicas_y_daso metricas_de_dos_comunidades_con_mezquite_Prosopis\%20laevigata_el_estado_de \%20Durango.pdf (2 de julio de 2021).

Vega C., J., A. Melgoza C. y J. S. Sierra T. 2006. Caracterización del crecimiento de dos especies de sotol (Dasylirion leiophyllum Engelm. ex Trelease y D. sereke Bogler) fertilizadas con nitrógeno y fósforo. Revista Mexicana de Ciencias Forestales 31(99): 55-71. http://cienciasforestales.inifap.gob.mx/editorial/index.php/forestales/article/view/840 (1 de agosto de 2021).

Velasco B., A. Arredondo G., M. C. Zamora-Martínez y F. Moreno S. 2009. Modelos predictivos para la producción de productos forestales no maderables: Lechuguilla. Cenid Comef, INIFAP. México, D.F. México. Folleto técnico No. 2. 56 p.

Villa-Castorena, M., E. A. Catalán-Valencia, J. G. Arreola-Ávila, M. A. Inzunza-Ibarra y A. Román L. 2011. Influencia de la frecuencia del riego en el crecimiento de orégano (Lippia graveolens HKB). Revista Chapingo Serie Ciencias Forestales y del Ambiente (17): 183-193. Doi: 10.5154/r.rchscfa.2010.10.088. 
Villanueva D., J., R. Jasso I., E. H. Cornejo O. y C. Potisek T. 2004. El mezquite en la comarca lagunera: su dinámica, volumen maderable y tasa de crecimiento anual. Revista Agrofaz 4(2): 633-648.

https://dialnet.unirioja.es/servlet/articulo?codigo=2302461 (4 de marzo de 2021).

Villavicencio G., E. E. y H. Franco L. 1992. Guía para la evaluación de existencias de palma samandoca (Yucca carnerosana Trel.) en el estado de Coahuila. CIRNEINIFAP, Campo Experimental Saltillo. Saltillo, Coah., México. Folleto técnico No. 2. 18 p. www.inifapcirne.gob.mx/Biblioteca/Publicaciones/40.pdf (4 de febrero de 2021).

Villavicencio G., E. E. 1993. Diagnostic on production and explotation of fiber. Fourth International Conference on Desert Development: Sustainable Development for our common Future. México D.F., México pp. 214-224.

www.drylanddevelop.org/uploads/6/1/7/8/61785389/4-.pdf (4 de febrero de 2021).

Villavicencio G., E. E., A. Villegas M., G. Arellano O. y J. Vargas H. 1999. Desarrollo de brotes in vitro de Astrophytum myriostigma Lem. Cactáceas y Suculentas Mexicanas 44(2): 49-57. https://biblat.unam.mx/es/revista/cactaceas-ysuculentas-mexicanas/9 (4 de febrero de 2021).

Villavicencio G., E. E., J. J. López G., O. U. Martínez B. y G. García P. 2006. Distribución digitalizada y características ecológicas del genero Ariocarpus spp. en Coahuila. CIRNE-INIFAP., Campo Experimental Saltillo. Saltillo, Coah., México. Publicación especial No. 8. 53 p. www.inifapcirne.gob.mx/Biblioteca/Publicaciones/203.pdf (4 de febrero de 2021).

Villavicencio G., E. E., A. Cano P. y A. Juárez S. 2007. Guía para la micropropagación y producción in vitro de plantas de sotol (Dasylirion cedrosanum Trel.). CIRNE-INIFAP, Campo Experimental Saltillo. Saltillo, Coah., México. Folleto técnico No. 37.29 p. www.inifapcirne.gob.mx/Biblioteca/Publicaciones/200.pdf (2 de enero de 2021). 
Villavicencio G., E. E., A. Cano P. y A. Juárez S. 2009. Micropropagación producción de plantas del bonete o birrete de obispo, cactácea ornamental amenazada de extinción del Desierto Chihuahuense. CIRNE-INIFAP, Campo Experimental Saltillo. Saltillo, Coah., México. Folleto técnico No. 39. 42 p.

www.gob.mx/cms/uploads/attachment/file/225108/Micropopagacion_y_produccion_ de_plantas_del_bonete_o_birrete_de_obispo.pdf (1 de enero de 2021).

Villavicencio G., E. E., A. Arredondo G., M. A. Carranza P., O. Mares A., S. Comparan S. y A. González C. 2010a. Cactáceas ornamentales del Desierto Chihuahuense que se distribuyen en Coahuila, San Luis Potosí y Nuevo León, México. CIRNE-INIFAP, Campo Experimental Saltillo. Saltillo, Coah., México. Libro técnico No. 2. 345 p. www.inifapcirne.gob.mx/Biblioteca/Publicaciones/846.pdf (8 de diciembre de 2020).

Villavicencio G., E. E., A. Cano P. y X. García C. 2010b. Metodología para determinar las existencias de orégano (Lippia graveolens H.B.K) en rodales naturales de Parras de la Fuente, Coahuila. CIRNE-INIFAP, Campo Experimental Saltillo. Saltillo, Coah., México. Folleto técnico No. 42. 42 p.

www.inifapcirne.gob.mx/Biblioteca/Publicaciones/850.pdf (8 de diciembre de 2020).

Villavicencio G., E. E., A. González C., A. Arredondo G., L. Iracheta D. J., S. Comparan S. y R. Casique V. 2011. Micropropagación de Turbinicarpus knuthianus (Boed.) John \& Riha cactacea ornamental del Desierto Chihuahuense, en estatus de riesgo. Revista Mexicana de Ciencias Forestales 2(6): 37-56. Doi: 10.29298/rmcf.v2i6.573.

Villavicencio G., E. E., M. A. Carranza P., S. Comparan S. y A. González C. 2012a. Micropropagación y producción de Turbinicarpus knuthianus (Boed.) John \& Riha cactácea ornamental del Desierto Chihuahuense. CIRNE-INIFAP, Campo Experimental Saltillo. Saltillo, Coah., México. Folleto técnico No. 48.41 p. www.inifapcirne.gob.mx/Biblioteca/Publicaciones/918.pdf ( 8 de enero de 2021). 
Villavicencio G., E. E., A. González C., M. A. Carranza P. y A. Arredondo G. 2012b. Micropropagación y producción de Epithelantha micromeris (Engelm.) F.A.C. Weber ex Britt. \& Rose cactácea ornamental del Desierto Chihuahuense. CIRNE-INIFAP, Campo Experimental Saltillo. Saltillo, Coah., México. Folleto técnico No. 51. 41 p. www.inifapcirne.gob.mx/Biblioteca/Publicaciones/921.pdf (4 de noviembre de 2020).

Villavicencio G., E. E., A. González C. y M. A. Carranza P. 2012c. Micropropagación de Epithelantha micromeris (Engelm.) F.A.C. Weber Ex Britt. \& Rose cactácea ornamental y recurso fitogenético del Desierto Chihuahuense. Revista Mexicana de Ciencias Forestales 3(14): 83-99. Doi: 10.29298/rmcf.v3i14.476.

Villavicencio G., E. E., M. A. Carranza P., A. González C., J. Valdés R., C. González H. y A. Arredondo G. 2013a. Guía técnica para la descripción varietal del chaute (Ariocarpus retusus Scheidw.) cactácea ornamental del Desierto Chihuahuense. CIRNE-INIFAP, Campo Experimental Saltillo. Saltillo, Coah., México. Folleto técnico No. 56. 52 p. www.inifapcirne.gob.mx/Biblioteca/Publicaciones/963.pdf (8 de enero de 2021).

Villavicencio G., E. E., M. A. Carranza P., A. González C., J. Valdés R., C. González H. y A. Arredondo G. 2013b. Manual gráfico para la descripción varietal del chaute (Ariocarpus retusus Scheidw.) cactácea ornamental del Desierto Chihuahuense. CIRNE-INIFAP, Campo Experimental Saltillo. Saltillo, Coah., México. Folleto técnico No. 57. 73 p. www.inifapcirne.gob.mx/Biblioteca/Publicaciones/962.pdf (1 de enero de 2021).

Villavicencio G., E. E., A. Hernández R., C. N. Aguilar G. y X. García C. 2018. Estimación de la biomasa foliar seca de Lippia graveolens Kunth del sureste de Coahuila. Revista Mexicana de Ciencias Forestales 9(45): 187-207. Doi: 10.29298/rmcf.v9i45.139.

Villavicencio G., E. E., L. I. Trejo T., L. Bañuelos H., M. A. López L. y G. Arellano O. 2019a. Nutrición mineral con nitrógeno, fósforo y potasio en la producción del barril azul en invernadero. Revista Mexicana Ciencias Agrícolas Pub. Esp. (23): 313-323. Doi: 10.29312 /remexca.v0i23.2030. 
Villavicencio-Gutiérrez, E. E., M. A. Carranza-Pérez y B. E. Zamora-Martínez. 2020a. Propagación y cultivo de cactus de ornato del Desierto Chihuahuense. CIRNE-INIFAP, Campo Experimental Saltillo. Saltillo, Coah., México. Folleto técnico Núm. 65. 67 p. https://vun.inifap.gob.mx/BibliotecaWeb/_Content?/=12301 (15 de marzo de 2021).

Villavicencio G., G. Arellano O. y V. A. Belmontes D. 2020b. Micropropagación del órgano pequeño sacasil (Echinocereus poselgeri Lem.) (Fam.: Cactaceae). Acta Horticulturae (1288): 123:130. Doi: 10.17660/ActaHortic.2020.1288.19.

Villavicencio-Gutiérrez E. E., S. Mendoza-Morales y J. Méndez-González. 2020c. Modelo para predecir biomasa foliar seca de Litsea parvifolia (Hemsl.) Revista Mexicana de Ciencias Forestales 11(58): 113-133. Doi: 29298/rmcf.v11i58.642.

Zamora-Martínez, M. C., C. Méndez E., R. Pérez M. y E. N. Cortés B. 2013. Euphorbia antisyphilitica Zucc.: recurso forestal no maderable de alto valor económico. Cenid Comef, INIFAP. México, D.F., México. Folleto técnico No. 12. 64 p. https://docplayer.es/58637473-Directorio-institucional-secretaria-de-agriculturaganaderia-desarrollo-rural-pesca-y-alimentacion-lic-enrique-martinez-martinezsecretario.html (3 de enero 2021).

\section{(c) (1) (9)}

Todos los textos publicados por la Revista Mexicana de Ciencias Forestales -sin excepción- se distribuyen amparados bajo la licencia Creative Commons 4.0 Atribución-No Comercial (CC BY-NC 4.0 Internacional), que permite a terceros utilizar lo publicado siempre que mencionen la autoría del trabajo y a la primera publicación en esta revista. 